\title{
Circulating B-vitamin biomarkers and B-vitamin supplement use in relation to quality of life in patients with colorectal cancer: results from the FOCUS consortium
}

Citation for published version (APA):

Koole, J. L., Bours, M. J. L., Geijsen, A. J. M. R., Gigic, B., Ulvik, A., Kok, D. E., Brezina, S., Ose, J., Baierl, A., Bohm, J., Brenner, H., Breukink, S. O., Chang-Claude, J., van Duijnhoven, F. J. B., van Duijvendijk, P., Gumpenberger, T., Habermann, N., van Halteren, H. K., Hoffmeister, M., ... Weijenberg, M. P. (2021). Circulating B-vitamin biomarkers and B-vitamin supplement use in relation to quality of life in patients with colorectal cancer: results from the FOCUS consortium. American Journal of Clinical Nutrition, 113(6), 1468-1481. https://doi.org/10.1093/ajcn/nqaa422

Document status and date:

Published: 01/06/2021

DOI:

10.1093/ajcn/nqaa422

Document Version:

Publisher's PDF, also known as Version of record

Document license:

Taverne

Please check the document version of this publication:

- A submitted manuscript is the version of the article upon submission and before peer-review. There can be important differences between the submitted version and the official published version of record. People interested in the research are advised to contact the author for the final version of the publication, or visit the DOI to the publisher's website.

- The final author version and the galley proof are versions of the publication after peer review.

- The final published version features the final layout of the paper including the volume, issue and page numbers.

Link to publication

\footnotetext{
General rights rights.

- You may freely distribute the URL identifying the publication in the public portal. please follow below link for the End User Agreement:

www.umlib.nl/taverne-license

Take down policy

If you believe that this document breaches copyright please contact us at:

repository@maastrichtuniversity.nl

providing details and we will investigate your claim.
}

Copyright and moral rights for the publications made accessible in the public portal are retained by the authors and/or other copyright owners and it is a condition of accessing publications that users recognise and abide by the legal requirements associated with these

- Users may download and print one copy of any publication from the public portal for the purpose of private study or research.

- You may not further distribute the material or use it for any profit-making activity or commercial gain

If the publication is distributed under the terms of Article 25fa of the Dutch Copyright Act, indicated by the "Taverne" license above, 


\title{
Circulating B-vitamin biomarkers and B-vitamin supplement use in relation to quality of life in patients with colorectal cancer: results from the FOCUS consortium
}

\begin{abstract}
Janna L Koole, ${ }^{1}$ Martijn JL Bours, ${ }^{1}$ Anne JMR Geijsen, ${ }^{2}$ Biljana Gigic, ${ }^{3}$ Arve Ulvik, ${ }^{4}$ Dieuwertje E Kok, ${ }^{2}$ Stefanie Brezina,${ }^{5}$ Jennifer Ose, ${ }^{6,7}$ Andreas Baierl, ${ }^{8}$ Jürgen Böhm, ${ }^{6,7}$ Hermann Brenner,,${ }^{9,10,11}$ Stéphanie O Breukink, ${ }^{12}$ Jenny Chang-Claude, ${ }^{13}$ Fränzel JB van Duijnhoven, ${ }^{2}$ Peter van Duijvendijk, ${ }^{14}$ Tanja Gumpenberger, ${ }^{5}$ Nina Habermann, ${ }^{15}$ Henk K van Halteren, ${ }^{16}$ Michael Hoffmeister, ${ }^{10}$ Andreana N Holowatyj, ${ }^{6,7,17,18}$ Maryska LG Janssen-Heijnen, ${ }^{1,19}$ Eric TP Keulen, ${ }^{20}$ Rama Kiblawi, ${ }^{6,7,21}$ Flip M Kruyt, ${ }^{22}$ Christopher I Li, ${ }^{23}$ Tengda Lin,${ }^{6,7}$ Øivind Midttun, ${ }^{4}$ Anita R Peoples, ${ }^{6,7}$ Eline H van Roekel, ${ }^{1}$ Martin A Schneider, ${ }^{3}$ Petra Schrotz-King, ${ }^{9}$ Alexis B Ulrich, ${ }^{3}$ Kathy Vickers, ${ }^{23}$ Evertine Wesselink, ${ }^{2}$ Johannes HW de Wilt, ${ }^{24}$ Andrea Gsur, ${ }^{5}$ Per M Ueland, ${ }^{4}$ Cornelia M Ulrich, ${ }^{6,7}$ Ellen Kampman, ${ }^{2}$ and Matty $P$ Weijenberg ${ }^{1}$
\end{abstract}

\footnotetext{
${ }^{1}$ Department of Epidemiology, GROW School for Oncology and Developmental Biology, Maastricht University, Maastricht, The Netherlands; ${ }^{2}$ Division of Human Nutrition and Health, Wageningen University \& Research, Wageningen, The Netherlands; ${ }^{3}$ Department of General, Visceral and Transplantation Surgery, University of Heidelberg, Heidelberg, Germany; ${ }^{4}$ BEVITAL, Bergen, Norway; ${ }^{5}$ Institute of Cancer Research, Department of Medicine I, Medical University of Vienna, Vienna, Austria; ${ }^{6}$ Huntsman Cancer Institute, Salt Lake City, UT, USA; ${ }^{7}$ Department of Population Health Sciences, University of Utah, Salt Lake City, UT, USA; ${ }^{8}$ Department of Statistics and Operations Research, University of Vienna, Vienna, Austria; ${ }^{9}$ Division of Preventive Oncology, National Center for Tumor Diseases and German Cancer Research Center, Heidelberg, Germany; ${ }^{10}$ Division of Clinical Epidemiology and Aging Research, German Cancer Research Center (DKFZ), Heidelberg, Germany; ${ }^{11}$ German Cancer Consortium (DKTK), German Cancer Research Center (DKFZ), Heidelberg, Germany; ${ }^{12}$ Department of Surgery, GROW School for Oncology and Developmental Biology, NUTRIM School of Nutrition and Translational Research in Metabolism, Maastricht University Medical Centre+, Mastricht, The Netherlands; ${ }^{13}$ Division of Cancer Epidemiology, German Cancer Research Center (DKFZ), Heidelberg, Germany; ${ }^{14}$ Department of Surgery, Gelre Hospital, Apeldoorn, The Netherlands; ${ }^{15}$ Genome Biology, European Molecular Biology Laboratory (EMBL), Heidelberg, Germany; ${ }^{16}$ Department of Internal Medicine, Admiraal de Ruyter Hospital, Goes, The Netherlands; ${ }^{17}$ Department of Medicine, Vanderbilt University Medical Center, Nashville, TN, USA; ${ }^{18}$ Vanderbilt-Ingram Cancer Center, Nashville, TN, USA; ${ }^{19}$ Department of Clinical Epidemiology, VieCuri Medical Centre, Venlo, The Netherlands; ${ }^{20}$ Department of Internal Medicine and Gastroenterology, Zuyderland Medical Centre, Sittard, The Netherlands; ${ }^{21}$ Medical Faculty, Ruprecht-Karls-University Heidelberg, Heidelberg, Germany; ${ }^{22}$ Department of Surgery, Gelderse Vallei Hospital, Ede, The Netherlands; ${ }^{23}$ Division of Public Health Sciences, Fred Hutchinson Cancer Research Center, Seattle, WA, USA; and ${ }^{24}$ Department of Surgery, Division of Surgical Oncology and Gastrointestinal Surgery, Radboud University Medical Center, Nijmegen, The Netherlands
}

\begin{abstract}
Background: B vitamins have been associated with the risk and progression of colorectal cancer (CRC), given their central roles in nucleotide synthesis and methylation, yet their association with quality of life in established CRC is unclear.

Objectives: To investigate whether quality of life 6 months postdiagnosis is associated with: 1) circulating concentrations of B vitamins and related biomarkers 6 months postdiagnosis; 2) changes in these concentrations between diagnosis and 6 months postdiagnosis; 3) B-vitamin supplement use 6 months postdiagnosis; and 4) changes in B-vitamin supplement use between diagnosis and 6 months postdiagnosis.

Methods: We included 1676 newly diagnosed stage I-III CRC patients from 3 prospective European cohorts. Circulating concentrations of 9 biomarkers related to the B vitamins folate, riboflavin, vitamin B6, and cobalamin were measured at diagnosis and 6 months postdiagnosis. Information on dietary supplement use was collected at both time points. Health-related quality of life (global quality of life, functioning scales, and fatigue) was assessed by the European Organisation for Research and Treatment of Cancer Quality of Life
\end{abstract}

Questionnaire 6 months postdiagnosis. Confounder-adjusted linear regression analyses were performed, adjusted for multiple testing. Results: Higher pyridoxal 5'-phosphate (PLP) was cross-sectionally associated with better physical, role, and social functioning, as well as reduced fatigue, 6 months postdiagnosis. Associations were observed for a doubling in the hydroxykynurenine ratio [3-hydroxykynurenine: (kynurenic acid + xanthurenic acid +3 hydroxyanthranilic acid + anthranilic acid); an inverse marker of vitamin B6] and both reduced global quality of life $(\beta=-3.62$; $95 \% \mathrm{CI}:-5.88,-1.36)$ and worse physical functioning $(\beta=$ -5.01 ; 95\% CI: $-7.09,-2.94)$. Dose-response relations were observed for PLP and quality of life. No associations were observed for changes in biomarker concentrations between diagnosis and 6 months. Participants who stopped using B-vitamin supplements after diagnosis reported higher fatigue than nonusers.

Conclusions: Higher vitamin B6 status was associated with better quality of life, yet limited associations were observed for the use of B-vitamin supplements. Vitamin B6 needs further study to clarify its role in relation to quality of life. Am J Clin Nutr 2021;113:14681481 . 
Keywords: colorectal cancer, B vitamins, dietary supplement use, health-related quality of life, fatigue, functioning, biomarkers, folates, vitamin B6, pyridoxal 5'-phosphate

\section{Introduction}

The diagnosis of colorectal cancer (CRC) imposes a major, long-term burden on a patient's physical, emotional, and social well-being (1). On average, $65 \%$ of CRC patients are alive 5 years after diagnosis, and the number of CRC survivors is expected to rise substantially in the coming decade (2-4).

Folates are of particular interest in relation to CRC. Folate may have a protective effect regarding the development of $\mathrm{CRC}$, whereas this vitamin could promote progression of already existing colorectal neoplasms $(5,6)$. Folate, together with other B vitamins, such as vitamin B2 (riboflavin), vitamin B6 [pyridoxal 5'-phosphate (PLP)], and vitamin B12 (cobalamin), are important factors in DNA synthesis and methylation reactions through

The COLON study (COlorectal cancer: Longitudinal, Observational study on Nutritional and lifestyle factors that influence colorectal tumour recurrence, survival and quality of life) was supported by Wereld Kanker Onderzoek Fonds (WKOF) and the World Cancer Research Fund International (WCRF International); the World Cancer Research Fund International Regular Grant Programme (WKOF/WCRF, the Netherlands, project number 2014/1179); Alpe d'Huzes/Dutch Cancer Society (KWF Kankerbestrijding, the Netherlands, project numbers UM 2012-5653, UW 2013-5927, and UW 2015-7946); ERA-NET (European Research Area Network) on Translational Cancer Research (TRANSCAN/Dutch Cancer Society, the Netherlands, project numbers UW 2013-6397 and UW 20146877); and the Netherlands Organization for Health Research and Development (ZonMw, the Netherlands). The EnCoRe study (Energy for life after ColoRectal cancer) was supported by grants from the Stichting Alpe d'HuZes within the research program "Leven met kanker" of the Dutch Cancer Society (grant numbers UM-2010-4867 and UM-2012-5653); grants from Kankeronderzoekfonds Limburg as part of Health Foundation Limburg (grant number 00005739); WKOF, as part of the WCRF International grant program (grant number 2016/1620); and ERA-NET on Translational Cancer Research (TRANSCAN/Dutch Cancer Society, the Netherlands, project number UM 2014-6877). ColoCare Heidelberg was funded by the ERANET on Translational Cancer Research (TRANSCAN; project 01KT1503; Federal Ministry of Education and Research); the National Cancer Institute (project R01 CA189184); the Stiftung Lebensblicke; and Matthias Lackas Foundation.

Supplemental Figure 1 and Supplemental Tables 1 and 2 are available from the "Supplementary data" link in the online posting of the article and from the same link in the online table of contents at https://academic.oup.com/ajen/.

EK and MPW contributed equally to this work.

Address correspondence to MPW (e-mail: mp.weijenberg@ maastrichtuniversity.nl).

Abbreviations used: AA, anthranilic acid; EnCoRe, Energy for life after ColoRectal cancer; COLON, COlorectal cancer: Longitudinal, Observational study on Nutritional and lifestyle factors that influence colorectal tumour recurrence, survival and quality of life; CRC, colorectal cancer; CRP, C-reactive protein; FDR, false discovery rate; FOCUS, biomarkers related to folatedependent 1-carbon metabolism in colorectal cancer recurrence and survival; HAA, 3'-hydroxyanthranilic acid; HK, 3-hydroxykynurenine; HKr, hydroxykynurenine ratio; hmTHF, 4- $\alpha$-hydroxy-5-methyltetrahydrofolate; MMA, methylmalonic acid; KA, kynurenic acid; KTR, kynurenine/tryptophan ratio; PLP, pyridoxal 5'-phosphate; tHcy, total homocysteine; XA, xanthurenic acid.

Received June 11, 2020. Accepted for publication December 14, 2020.

First published online March 1, 2021; doi: https://doi.org/10.1093/ajcn/ nqaa422. a network of interdependent metabolic pathways that mediate the transfer of 1-carbon units $(5,6)$. It is unknown whether B vitamins may play a role in reducing health problems among CRC survivors.

Fatigue is highly prevalent during anticancer therapy and generally decreases after treatment completion (7). However, between $40 \%$ and $50 \%$ of CRC survivors still experience fatigue in the first year following diagnosis $(8,9)$. Folic acid and vitamin B12 supplementation may be effective in treating chronic fatigue syndrome in case of deficiencies (10), although an intervention study in patients with irritable bowel syndrome or inflammatory bowel disease found no effect of vitamin B12 supplementation on fatigue (11). B vitamins may further be effective in improving cognitive functioning, as elevated homocysteine-a marker of folate and B12 deficiency-is associated with neuropsychiatric diseases (12). Nonetheless, intervention studies investigating the effects of folic acid, vitamin B6, and vitamin B12 supplement use, alone or combined, on cognitive functioning in elderly populations were not conclusive (12-14). Circulating unmetabolized folic acid has been associated with adverse effects, including impaired cognitive functions, in the elderly $(15,16)$. Further, higher homocysteine concentrations have prospectively been associated with a decline in physical functioning in older adults $(17,18)$. Elevated homocysteine and low folate concentrations have been associated with the etiology of psychiatric problems (19).

Dietary supplements are used by $20-85 \%$ of cancer survivors, yet their benefits remain uncertain $(9,20,21)$. Investigating whether B-vitamin status and the use of B-vitamin supplements are associated with quality of life after CRC diagnosis is a first step in evaluating whether B vitamins may be beneficial for quality of life. Additional insight may be obtained by investigating whether an increase or decrease in biomarker concentrations between diagnosis and 6 months postdiagnosis and the initiation or discontinuation of B-vitamin supplement use could influence quality of life.

The current study aims to investigate whether quality of life at 6 months postdiagnosis is associated with: 1) circulating concentrations of $\mathrm{B}$ vitamins and related biomarkers 6 months postdiagnosis; 2) changes in these concentrations between diagnosis and 6 months postdiagnosis; 3) B-vitamin supplement use 6 months postdiagnosis; and 4) changes in B-vitamin supplement use between diagnosis and 6 months postdiagnosis.

\section{Methods}

\section{The FOCUS consortium}

Data were analyzed from 3 prospective cohort studies participating in the "biomarkers related to folate-dependent 1carbon metabolism in colorectal cancer recurrence and survival" (FOCUS) consortium. The primary objective of the FOCUS consortium is to evaluate whether folate status at diagnosis and changes during and after treatment are related to prognoses of stage I-III CRC patients. Studies included in the current analyses are the COLON study (COlorectal cancer: Longitudinal, Observational study on Nutritional and lifestyle factors that influence colorectal tumour recurrence, survival and quality of life), from Wageningen University \& Research, the Netherlands (registered at clinicalTrials.gov as 


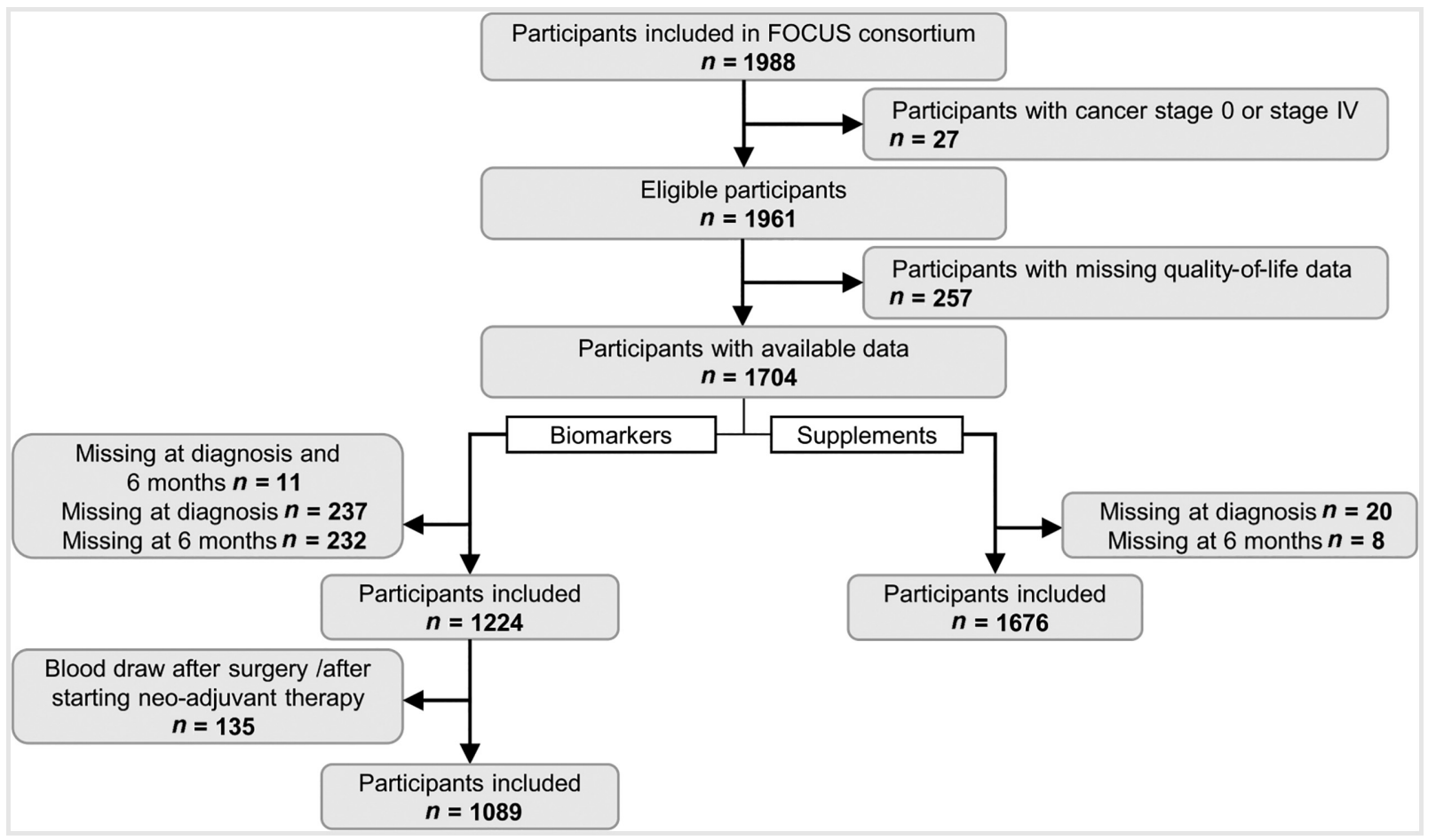

FIGURE 1 Flow diagram of the inclusion of participants in the analyses for (A) biomarker concentrations and (B) B-vitamin supplement use with healthrelated quality of life in colorectal cancer patients from the FOCUS consortium, for the cohorts with data relevant for the research question of this paper. Selections were made based on the availability of blood samples to measure biomarker concentrations and the availability of information on B-vitamin supplement use. The overlap between both data sets was 1077 participants (64\%). Abbreviation: FOCUS, biomarkers related to folate-dependent 1-carbon metabolism in colorectal cancer recurrence and survival.

NCT03191110) (22); the EnCoRe study (Energy for life after ColoRectal cancer), from Maastricht University, the Netherlands (registered at the Netherlands Trial Register as NL6904) (23); and the Heidelberg site of the ColoCare study, from the National Center for Tumor Diseases in Heidelberg, Germany (registered at clinicaltrials.gov as NCT02328677) (24). Newly diagnosed CRC patients aged $>18$ years were recruited upon diagnosis. Each study was approved by local ethics committees, and all participants signed informed consent.

The current analyses included only patients with cancer stages I-III. A first baseline measurement took place shortly after diagnosis, prior to the start of treatment (COLON and EnCoRe) or prior to undergoing surgery (ColoCare). Patients $(n=135)$ were excluded from the analyses on biomarker concentrations when the baseline blood draw took place after the start of neoadjuvant therapy. A follow-up measurement took place 6 months after baseline (COLON and ColoCare) or after the treatment end date (EnCoRe). As the EnCoRe study includes an additional follow-up measurement at 6 weeks after the treatment end date, either the 6 week or 6 month posttreatment measurement was selected for these analyses, based on the measurement that was closest to the time point 6 months postdiagnosis. In this way, timing of the measurements was comparable between studies.

Participants were included in the current analyses based on the availability of data (Figure 1). Information on the independent variables, such as biomarker concentrations or Bvitamin supplement use, was required to be available both at diagnosis and at 6 months after diagnosis in order to adjust for baseline biomarker concentrations and calculate changes between the time points. Ultimately, a total of 1089 participants were included for the analyses on biomarker concentrations and 1676 patients were included for the analyses on B-vitamin supplement use.

\section{Blood biomarkers}

EDTA plasma (EnCoRe and COLON) or serum (ColoCare) samples were collected, divided into aliquots, and stored at $-80{ }^{\circ} \mathrm{C}$ until analysis. Samples were shipped on dry ice to the laboratory of Bevital in Bergen, Norway (www.bevital.no). Available samples were shipped in 2 batches with a 1-year time interval from 2016-2018 to increase the final number of samples to be included for the analyses. ColoCare sample volumes were limited, and therefore diluted (1:2). The dilution factor was taken into account by Bevital when analyzing the samples.

Nine principal biomarkers related to the folate-dependent 1carbon metabolism were examined. Total folate was analyzed as the sum of 5-methyltetrahydrofolate and 4- $\alpha$-hydroxy-5methyltetrahydrofolate (hmTHF) (25). Folic acid, the synthetic form of folate, was also analyzed. Total homocysteine (tHcy) was included as a marker of folate and vitamin B12 status. Methylmalonic acid (MMA) was analyzed alongside cobalamin as a functional marker of vitamin B12 status. Methionine was included, given its close connection with homocysteine and cobalamin in the methylation cycle. Riboflavin was included as 
a marker of vitamin B2 status. PLP was included as the most commonly used marker of vitamin B6 status, along with the hydroxykynurenine ratio $(\mathrm{HKr})$, a recently described functional marker of vitamin B6 status calculated as the ratio of 3hydroxykynurenine (HK) to the sum of kynurenic acid (KA), xanthurenic acid (XA), 3'-hydroxyanthranilic acid (HAA), and anthranilic acid (AA) (26).

LC-MS/MS was used to analyze the folate species mTHF, hmTHF, and folic acid (27); the tryptophan metabolites HK, KA, $\mathrm{XA}, \mathrm{HAA}$, and AA; and riboflavin, PLP, cotinine, and creatinine $(28,29)$. The biomarkers kynurenine, tryptophan, tHcy, methionine, and MMA were analyzed by gas chromatography-MS/MS (30). Cobalamin was measured by a microbiological assay (31), and C-reactive protein (CRP) was measured by an immunomatrix-assisted laser desorption/ionization-MS approach (32).

Duplicate samples with known biomarker concentrations, as well as calibrator samples and a blank vial, were added to each assay tray by BEVITAL to control for quality of the samples. Additionally, each cohort provided duplicate samples to be included on every plate. The coefficients of variation from a total of 160 duplicate samples ranged from $1.8 \%$ for cobalamin to $12.2 \%$ for hmTHF.

\section{Use of supplements containing B vitamins}

Information on the use of supplements containing $\mathrm{B}$ vitamins was collected by self-administered questionnaires (COLON and ColoCare) or interviews by research dieticians during home visits (EnCoRe). The COLON questionnaire contained questions on the intake of multivitamins, B-vitamin complexes, and single vitamin B12 and folic acid use, and was developed by the Division of Human Nutrition and Health, Wageningen University \& Research (22). When multivitamins were used, participants were considered to be using B vitamins. Within the EnCoRe study, participants were asked about the supplements they were using, and were asked to show the original package when available. All details on (brand) name and frequency were listed on standardized registration forms. In ColoCare, detailed selfreported questionnaires were used to collect data on regular dietary supplement use. The intake of multivitamins, 11 single vitamins, 6 minerals, and 14 other ingredients (e.g., herbal supplements), as well as frequencies of intakes, were recorded prior to surgery and 6 months thereafter. The reference period for supplement use at diagnosis was either the preceding year (COLON and EnCoRe) or the preceding month (ColoCare). At 6 months postdiagnosis, reference periods were the preceding 6 months (COLON and ColoCare) or the period since the previous measurement (EnCoRe) that took place either at diagnosis (mean of 6.5 preceding months) or at 6 weeks posttreatment (mean of 4.5 preceding months), because the EnCoRe study included an additional follow-up measurement at 6 weeks after the treatment end date.

For the current analyses, the frequency of B-vitamin supplement use was defined as use of any dietary supplement (including multivitamins) containing at least 1 of the B vitamins (folate/folic acid, riboflavin, vitamin B6, or vitamin B12) at least once per week, for a consecutive period of 1 month or more. Details on the dosages of B-vitamin intakes were not considered in the current analyses. B-vitamin supplement use was categorized into 4 mutually exclusive classes: 1) participants who started use after diagnosis; 2) participants who stopped use after diagnosis; 3) nonusers at both diagnosis and 6 months after diagnosis; and 4) users at both diagnosis and 6 months after diagnosis.

\section{Health-related quality of life}

At 6 months after diagnosis, all studies assessed health-related quality of life using the European Organisation for Research and Treatment of Cancer Quality of Life Questionnaire (QLQC30, version 3.0), a 30-item validated questionnaire $(33,34)$. The subscale for global quality of life was analyzed, as well as 5 functional scales (i.e., physical, role, emotional, cognitive, and social functioning) and the symptom scale for fatigue. All scales range from $0-100$, and a higher score indicates better quality of life and functioning, except for in the fatigue scale, where higher scores are indicative of more fatigue. Previously published thresholds for clinical importance were used to interpret the scores (35).

\section{Measurement of covariates}

Information on cancer stage, therapy, and other clinical variables were derived from medical records or clinical registries. In order to calculate BMI $\left(\mathrm{kg} / \mathrm{m}^{2}\right)$, height and weight were measured during home visits (EnCoRe), were self-reported (COLON), or were taken from anesthesia protocols (ColoCare). Adherence to the physical activity guideline to spend at least 150 minutes per week in moderate-to-vigorous physical activity was assessed based on self-report, either using the Short Questionnaire to Assess Health-enhancing physical activity questionnaire (COLON and EnCoRe) $(22,23)$ or the VITamins And Lifestyle cohort study questionnaire (ColoCare) (24). Dietary information at 6 months postdiagnosis (i.e., total energy and alcohol intakes) was assessed using an FFQ by COLON and ColoCare and a 7-day dietary record by EnCoRe. Cotinine and creatinine concentrations were included in the models to adjust for nicotine exposure and kidney functions, respectively, and were part of the biomarker analyses as measured at Bevital.

\section{Statistical methods}

Descriptive statistics were presented for the characteristics of the total study population and the individual cohorts, using means and SDs for normally distributed variables, medians and IQRs for skewed variables, and frequencies for categorical variables. In line with the 4 objectives of the study, 4 main analyses were performed.

Multivariable linear regression models were used to investigate the associations of biomarkers with health-related quality of life in cross-sectional analyses at 6 months postdiagnosis. Biomarker concentrations were $\log 2$-transformed, and the regression coefficients can therefore be interpreted as effects of doubling in concentrations.

A multivariable linear regression analysis was used to analyze changes in biomarker concentrations between diagnosis and 6 months with health-related quality of life at 6 months postdiagnosis. Changes were calculated by subtracting the concentration at diagnosis from the concentration at 6 months. Subsequently, individual change scores were z-standardized by subtracting the estimated population mean change and dividing by the estimated population SD of change scores, in order to be able to compare the magnitudes of changes across the biomarkers. 
Change scores were normally distributed, and therefore not transformed.

B-vitamin supplement use was modeled as a binary variable in cross-sectional analyses (use at 6 months: yes/no).

Changes in B-vitamin supplement use were modeled for the 4 different classes of supplement users and performed with 3 indicator variables, with nonuse at every time point as the reference category.

An a priori selected set of confounders was used, based on the literature and their hypothesized relations with the exposure and outcome variables. The analyses on biomarker concentrations were adjusted for the confounders sex, age, cohort (COLON, EnCoRe, ColoCare), neo-adjuvant therapy (yes/no), adjuvant therapy (yes/no), cancer stage (I, II, III), any supplement use (yes/no), total energy intake (kcal/day), alcohol intake (g/day), BMI $\left(\mathrm{kg} / \mathrm{m}^{2}\right)$, physical activity (adherence to international guidelines yes/no), cotinine (nmol/L), and creatinine $(\mu \mathrm{mol} / \mathrm{L})$. Adjustment for any supplement use was considered necessary because the use of supplements in general has previously been associated with a low quality of life and increased fatigue (9, 36). Analyses of changes in biomarker concentrations were additionally adjusted for the concentration at diagnosis.

Analyses on B-vitamin supplement use were adjusted for sex, age, cohort (COLON, EnCoRe, ColoCare), neo-adjuvant therapy (yes/no), adjuvant therapy (yes/no), cancer stage (I, II, III), total energy intake ( $\mathrm{kcal} / \mathrm{day}), \mathrm{BMI}\left(\mathrm{kg} / \mathrm{m}^{2}\right)$, physical activity (adherence to national guidelines yes/no), and any supplement use (yes/no). Any supplement use was added to the model for the aforementioned reason.

The cross-sectional analyses of biomarkers and supplement use at 6 months after diagnosis were adjusted for all abovementioned covariates measured at 6 months after diagnosis. In the analyses of changes, covariates were added as the change between diagnosis and 6 months for BMI, cotinine, and creatinine. Other covariates were either not available at diagnosis for all cohorts (e.g., dietary variables) or not considered relevant to include as changes (e.g., age), and therefore were added as measured at 6 months after diagnosis.

Since many different exposures were associated with many different outcomes, the analyses for each of the 4 objectives were separately adjusted for multiple testing using the false discovery rate (FDR) method (37).

Subanalyses were performed to evaluate the influence of outliers on the analyses. Log2-transformed biomarker concentrations with values 3 SDs below or above the mean within each cohort were excluded and compared to the original analyses to evaluate their influence. Sensitivity analyses were done by stratification by cohort to evaluate associations within each cohort separately. Further, given the relationship between B vitamins and inflammation, the inflammatory markers CRP and kynurenine/tryptophan ratio (KTR) were used to evaluate potential confounding by inflammation when statistically significant associations were observed $(38,39)$. In addition, Bvitamin concentrations were modeled as index variables by categories in relation to quality of life to explore potential doseresponse relationships, only performed when associations were still statistically significant after adjustment for multiple testing. Deficient, suboptimal, and adequate levels of vitamin B6 were defined as serum/plasma PLP concentrations of $<20 \mathrm{nmol} / \mathrm{L}, 20$ $30 \mathrm{nmol} / \mathrm{L}$, and $>30 \mathrm{nmol} / \mathrm{L}$, respectively (40).
Analyses were performed in $\mathrm{R}$, version 3.6.1 ( $\mathrm{R}$ Studio). Statistical tests with $P$ values below 0.05 after adjustment for multiple testing were considered statistically significant.

\section{Results}

Table 1 presents the characteristics of study participants $(n=1676)$ included in the analyses for the associations between B-vitamin supplement use and health-related quality of life. In general, characteristics of the 1089 participants included in the analyses on biomarker concentrations were comparable to the characteristics presented in Table 1; the overlap between both data sets was 1077 participants (64\%).

On average, $62 \%$ of the study population were colon cancer patients and $36 \%$ were rectum/rectosigmoid cancer patients. ColoCare included a larger proportion of rectum/rectosigmoid cancer patients as compared to COLON and EnCoRe. Onefourth $(26 \%)$ of participants were diagnosed with stage I CRC, $30 \%$ with stage II CRC, and $41 \%$ with stage III CRC. ColoCare participants were more commonly diagnosed with stage II CRC (41\%) as compared to EnCoRe (23\%) and COLON (29\%) participants. In total, $96 \%$ of patients received surgery, $24 \%$ received neo-adjuvant therapy, and $26 \%$ received adjuvant therapy.

Supplements containing B vitamins were used by $24 \%$ of all participants at diagnosis and by $19 \%$ of participants after 6 months (Table 1). B-vitamin supplement use was considerably lower for ColoCare participants as compared to the Dutch cohorts. Further differences between the cohorts could be observed in lifestyle-related variables, such as BMI, adherence to physical activity guidelines, and total energy and alcohol intakes. Scoring on all subscales of health-related quality of life was lower for ColoCare as compared to EnCoRe and COLON (Table 1). Furthermore, $44 \%$ of all participants experienced clinically important impairment in physical functioning, with between $20 \%$ and $30 \%$ experiencing impairments in role, emotional, and cognitive functioning and fatigue and $16 \%$ experiencing impairments in social functioning.

Minimal changes were observed in biomarker concentrations between baseline and 6 months after diagnosis in the total population (Table 2). Spearman correlations between the separate biomarkers were similar at the 2 time points and demonstrated expected correlations, including (at diagnosis) correlations of -0.46 for PLP with $\mathrm{HKr},-0.30$ for folate with tHcy, -0.29 for cobalamin with tHcy, and -0.27 for cobalamin with MMA (Supplementary Figure 1).

When biomarker concentrations were stratified by the 4 types of supplement users, the highest concentrations of folate, riboflavin, PLP, and cobalamin were observed at each time point for users of B-vitamin supplements and the lowest concentrations were observed for nonusers (Supplementary Table 1). As could be anticipated, mean concentrations of participants who started using B-vitamin supplements showed an increase from diagnosis to 6 months postdiagnosis, whereas mean concentrations of participants who stopped using B-vitamin supplements after diagnosis showed a decrease towards the 6-month time point. 
TABLE 1 Characteristics of study participants included in the FOCUS consortium, for the total population and stratified by cohort

\begin{tabular}{|c|c|c|c|c|}
\hline Participant characteristics $^{1}$ & Total population ${ }^{2}$ & COLON & EnCoRe & ColoCare \\
\hline Number of participants, $n(\%)$ & 1676 & 1200 (71.6) & $274(16.4)$ & $202(12.1)$ \\
\hline Men, $n(\%)$ & $1068(63.8)$ & $758(63.2)$ & $184(67.7)$ & $126(62.4)$ \\
\hline Age at diagnosis in years, mean (SD) & $65.6(9)$ & $65.8(8.6)$ & $66.5(9.4)$ & $63.3(11.8)$ \\
\hline \multicolumn{5}{|l|}{ Clinical characteristics } \\
\hline \multicolumn{5}{|l|}{ Tumor location, $n(\%)$} \\
\hline Colon & $1037(61.9)$ & 779 (64.9) & $163(59.5)$ & $95(47.0)$ \\
\hline Rectum/Rectosigmoid & $606(36.2)$ & $390(32.5)$ & $109(39.8)$ & $107(53.0)$ \\
\hline Unknown/Missing & $33(2.0)$ & $31(2.6)$ & $2(0.7)$ & 0 \\
\hline \multicolumn{5}{|l|}{ Cancer stage, $n(\%)$} \\
\hline I & $429(25.6)$ & $294(24.5)$ & $79(28.8)$ & $56(27.7)$ \\
\hline II & $496(29.6)$ & $350(29.2)$ & $63(23.0)$ & $83(41.1)$ \\
\hline III & $684(40.8)$ & $502(41.8)$ & $119(43.4)$ & $63(31.2)$ \\
\hline Unspecified/Unknown & $67(4.0)$ & $54(4.5)$ & $13(4.7)$ & 0 \\
\hline \multicolumn{5}{|l|}{ Surgery, $n(\%)$} \\
\hline Yes & $1606(95.8)$ & 1159 (96.6) & $245(89.4)$ & $202(100.0)$ \\
\hline No & $35(2.1)$ & $8(0.7)$ & $27(9.9)$ & 0 \\
\hline Unknown/Missing & $35(2.1)$ & $33(2.8)$ & $2(0.7)$ & 0 \\
\hline \multicolumn{5}{|l|}{ Neo-adjuvant therapy, $n(\%)$} \\
\hline Yes & $399(23.8)$ & $269(22.4)$ & $76(27.4)$ & $54(26.7)$ \\
\hline No & $1242(74.1)$ & $899(74.9)$ & $196(71.5)$ & $147(72.8)$ \\
\hline Unknown/Missing & $35(2.1)$ & $32(2.7)$ & $2(0.7)$ & $1(0.5)$ \\
\hline \multicolumn{5}{|l|}{ Adjuvant therapy, $n(\%)$} \\
\hline Yes & $434(25.9)$ & $284(23.7)$ & $78(28.5)$ & $72(35.6)$ \\
\hline No & $1147(68.4)$ & $828(69.0)$ & $194(70.8)$ & $125(61.9)$ \\
\hline Unknown/Missing & $95(5.7)$ & $88(7.3)$ & $2(0.7)$ & $5(2.5)$ \\
\hline \multicolumn{5}{|l|}{ Lifestyle characteristics } \\
\hline $\mathrm{BMI}$ in $\mathrm{kg} / \mathrm{m}^{2}$, mean $\pm \mathrm{SD}$ & $26.5 \pm 4.0$ & $26.3 \pm 3.9$ & $28.1 \pm 4.5$ & $25.6 \pm 3.8$ \\
\hline Adherence to physical activity guidelines, ${ }^{3} n(\%)$ & $990(60.6)$ & $766(65.6)$ & $169(63.3)$ & $55(27.4)$ \\
\hline Any supplement use, $n(\%)$ & $625(37.3)$ & 487 (40.6) & $90(32.9)$ & $48(23.8)$ \\
\hline Total energy intake ${ }^{4}$ in kcal/day, mean (SD) & $1900 \pm 565$ & $1816 \pm 501$ & $2062 \pm 487$ & $2317 \pm 888$ \\
\hline Alcohol intake $e^{4}$ in gr/day, median (IQR) & $5.2(0.3-17.5)$ & $4.8(0.1-16.5)$ & $5.7(0.0-21.0)$ & $9.0(1.9-24.3)$ \\
\hline Cotinine $^{5}$ in $n m o l / L$, mean $\pm S D$ & $90 \pm 320$ & $84 \pm 300$ & $101 \pm 366$ & $117 \pm 378$ \\
\hline Creatinine $^{5}$ in $\mu \mathrm{mol} / \mathrm{L}$, mean $\pm \mathrm{SD}$ & $82.4 \pm 18.9$ & $81.1 \pm 17.4$ & $85.2 \pm 21.6$ & $87.2 \pm 23.8$ \\
\hline \multicolumn{5}{|l|}{ Use of B vitamin-containing supplements ${ }^{6}$} \\
\hline At diagnosis, $n(\%)$ & $402(24.0)$ & $318(26.5)$ & $63(23.0)$ & $21(10.4)$ \\
\hline 6 months postdiagnosis, $n(\%)$ & $323(19.3)$ & $259(21.6)$ & $54(19.7)$ & $10(5.0)$ \\
\hline Users at diagnosis and 6 months, $n(\%)$ & $232(13.8)$ & $186(15.5)$ & $42(15.3)$ & $4(2.0)$ \\
\hline Participants who started use after diagnosis, $n(\%)$ & $91(5.4)$ & $73(6.1)$ & $12(4.4)$ & $6(3.0)$ \\
\hline Participants who stopped use after diagnosis, $n(\%)$ & $167(10.0)$ & $129(10.8)$ & $21(7.7)$ & $17(8.4)$ \\
\hline Nonusers at diagnosis and 6 months, $n(\%)$ & $1173(70.0)$ & $801(66.8)$ & $197(71.9)$ & $175(86.6)$ \\
\hline \multicolumn{5}{|l|}{ Health-related quality of life ${ }^{7}$} \\
\hline \multicolumn{5}{|l|}{ Global quality of life $e^{8}$} \\
\hline Scoring, mean $\pm \mathrm{SD}$ & $72.8 \pm 20.0$ & $74.6 \pm 19.0$ & $75.5 \pm 19.2$ & $58.2 \pm 20.5$ \\
\hline \multicolumn{5}{|l|}{ Physical functioning } \\
\hline Scoring, mean $\pm \mathrm{SD}$ & $80.7 \pm 18.9$ & $82.2 \pm 18.0$ & $79.3 \pm 19.0$ & $74.1 \pm 22.0$ \\
\hline $\mathrm{TCI} \leq 83, n(\%)$ & $742(44.3)$ & 497 (41.4) & $129(47.1)$ & $116(57.4)$ \\
\hline \multicolumn{5}{|l|}{ Role functioning } \\
\hline Scoring, mean $\pm \mathrm{SD}$ & $73.3 \pm 29.6$ & $74.9 \pm 29.2$ & $75.2 \pm 27.6$ & $61.3 \pm 31.7$ \\
\hline $\mathrm{TCI} \leq 58, n(\%)$ & $418(24.9)$ & $282(23.5)$ & $56(20.4)$ & $80(39.6)$ \\
\hline \multicolumn{5}{|l|}{ Emotional functioning } \\
\hline Scoring, mean $\pm \mathrm{SD}$ & $83.3 \pm 20.2$ & $85.8 \pm 17.5$ & $87.3 \pm 18.1$ & $63.1 \pm 26.0$ \\
\hline $\mathrm{TCI} \leq 71, n(\%)$ & $389(23.2)$ & $230(19.2)$ & 45 (16.4) & $114(56.4)$ \\
\hline \multicolumn{5}{|l|}{ Cognitive functioning } \\
\hline Scoring, mean $\pm \mathrm{SD}$ & $84.7 \pm 20.0$ & $85.4 \pm 19.0$ & $85.8 \pm 20.1$ & $78.6 \pm 24.0$ \\
\hline $\mathrm{TCI} \leq 75, n(\%)$ & $422(25.2)$ & $288(24.0)$ & $58(21.2)$ & $76(37.6)$ \\
\hline \multicolumn{5}{|l|}{ Social functioning } \\
\hline Scoring, mean $\pm \mathrm{SD}$ & $80.8 \pm 24.1$ & $82.6 \pm 22.3$ & $84.4 \pm 21.6$ & $65.5 \pm 31.0$ \\
\hline $\mathrm{TCI} \leq 58, n(\%)$ & $268(16.0)$ & $159(13.3)$ & 35 (12.8) & 74 (36.6) \\
\hline \multicolumn{5}{|l|}{ Fatigue } \\
\hline Scoring, mean $\pm \mathrm{SD}$ & $29.0 \pm 25.7$ & $27.6 \pm 25.2$ & $26.1 \pm 22.4$ & $41.3 \pm 29.0$ \\
\hline $\mathrm{TCI} \geq 39, n(\%)$ & $477(28.5)$ & $319(26.6)$ & $61(22.3)$ & $97(48.0)$ \\
\hline
\end{tabular}

Abbreviations: COLON, COlorectal cancer: Longitudinal, Observational study on Nutritional and lifestyle factors that influence colorectal tumour recurrence, survival and quality of life; EnCoRe, Energy for life after ColoRectal cancer; FOCUS, biomarkers related to folate-dependent 1-carbon metabolism in colorectal cancer recurrence and survival; TCI, threshold for clinical importance.

${ }^{1}$ Characteristics are given for the time point 6 months posttreatment, except when stated otherwise.

${ }^{2}$ Data are for the 1676 participants included in the analyses for B-vitamin supplement use and health-related quality of life. Participant characteristics of the 1089 participants included in the analyses on biomarker concentrations were comparable to the characteristics presented here. The overlap between both data sets was 1077 participants (64\%).

${ }^{3}$ Moderate-to-vigorous physical activity of at least 150 minutes per week.

${ }^{4}$ Total energy and alcohol intakes were assessed using a FFQ by COLON and ColoCare and by 7-day dietary records by the EnCoRe cohort.

${ }^{5}$ Cotinine and creatinine concentrations were included as markers of nicotine exposure and kidney function, respectively, and were measured in plasma for EnCoRe and COLON and in serum for ColoCare.

${ }^{6} \mathrm{~B}$-vitamin supplement use was defined as the use of dietary supplements containing at least 1 of the B vitamins: folic acid, vitamin B2, vitamin B6, or vitamin B12.

${ }^{7}$ Ranges of the European Organisation for Research and Treatment of Cancer Quality of Life Questionnaire subscales: 0-100. Higher scores are indicative of better health-related quality of life, except for fatigue, where higher scoring is indicative of more fatigue.

${ }^{8}$ No threshold for clinical importance was available. 


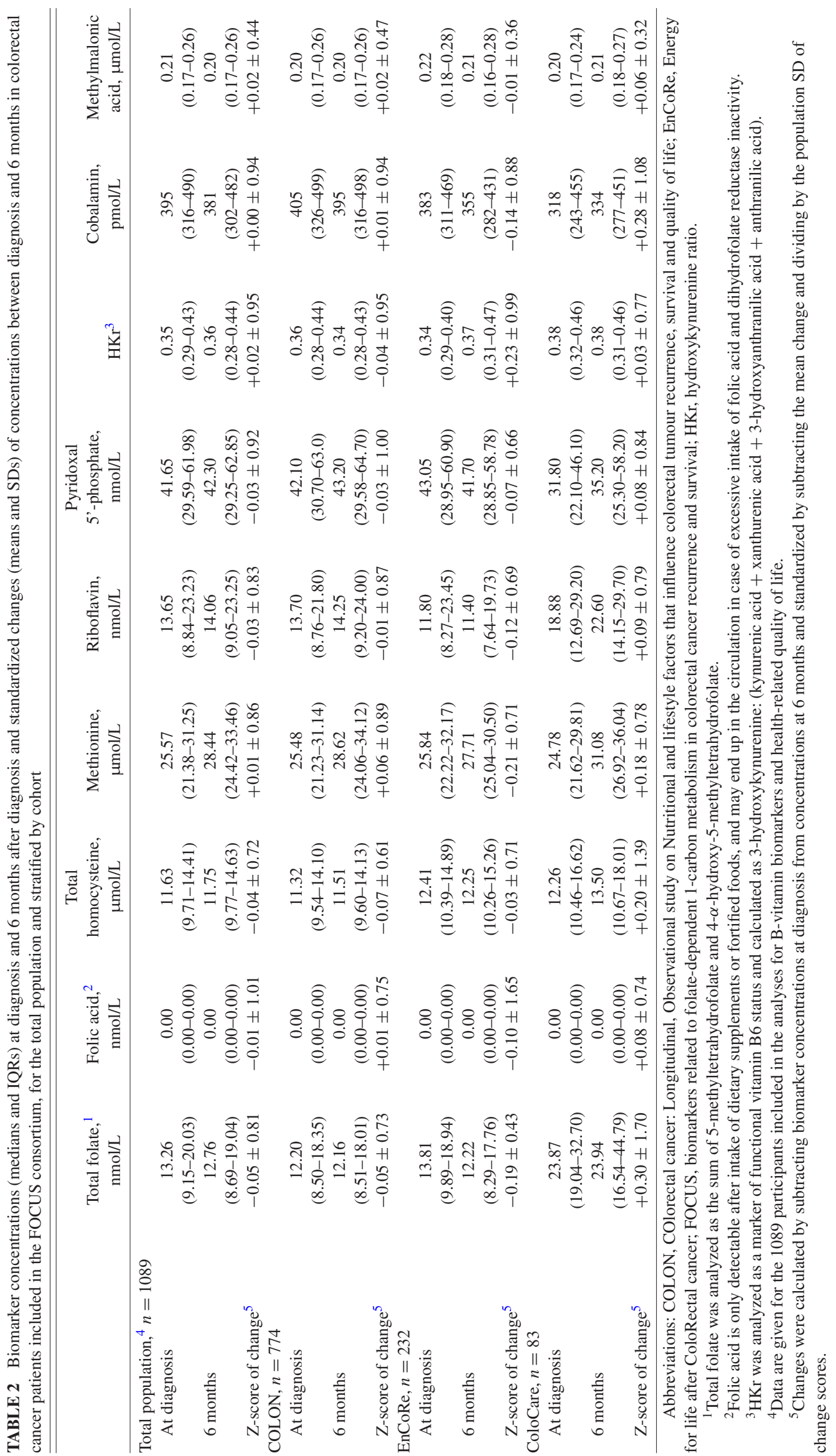


Global Quality of Life

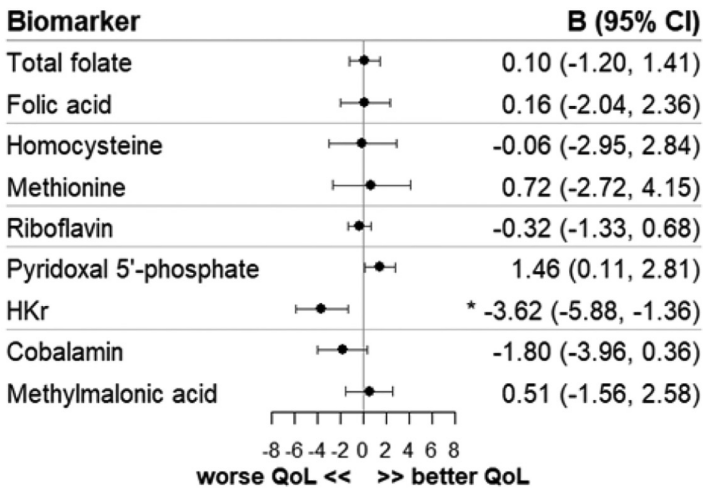

\section{Physical functioning}

Biomarker

\begin{tabular}{|c|c|c|c|}
\hline Biomarker & & & $B(95 \%$ Cl) \\
\hline Total folate & \multicolumn{2}{|c|}{$\mapsto-1$} & $0.01(-1.20,1.22)$ \\
\hline Folic acid & \multicolumn{2}{|c|}{$\bullet$} & $1.09(-1.18,3.37)$ \\
\hline Homocysteine & \multirow[t]{2}{*}{$\longmapsto$} & \multirow[b]{2}{*}{$\longmapsto$} & $-3.20(-5.87,-0.52)$ \\
\hline Methionine & & & $4.29(1.12,7.47)$ \\
\hline Riboflavin & \multicolumn{2}{|c|}{ 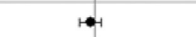 } & $-0.36(-1.29,0.57)$ \\
\hline Pyridoxal 5'-phosphate & & \multirow[t]{2}{*}{$\mapsto \bullet$} & * $2.55(1.31,3.80)$ \\
\hline $\mathrm{HKr}$ & $\mapsto$ & & ${ }^{\star}-5.01(-7.09,-2.94)$ \\
\hline Cobalamin & \multicolumn{2}{|c|}{$\mapsto$} & $-1.23(-3.23,0.77)$ \\
\hline Methylmalonic acid & \multicolumn{2}{|c|}{ 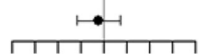 } & $-0.45(-2.37,1.47)$ \\
\hline
\end{tabular}

Cognitive functioning

\begin{tabular}{|c|c|c|}
\hline Biomarker & & B $(95 \% \mathrm{Cl})$ \\
\hline Total folate & $\mapsto-1$ & $0.20(-1.16,1.56)$ \\
\hline Folic acid & $\bullet$ & $1.18(-0.83,3.19)$ \\
\hline Homocysteine & $\longmapsto$ & $0.75(-2.27,3.78)$ \\
\hline Methionine & $\longmapsto$ & $0.21(-3.38,3.79)$ \\
\hline Riboflavin & 1 & $0.32(-0.73,1.37)$ \\
\hline Pyridoxal 5'-phosphate & $-\rightarrow-1$ & $1.37(-0.04,2.79)$ \\
\hline $\mathrm{HKr}$ & $\mapsto$ & $-1.43(-3.81,0.95)$ \\
\hline Cobalamin & $\mapsto$ & $-2.08(-4.31,0.16)$ \\
\hline Methylmalonic acid & $\mapsto \bullet$ & $-0.60(-2.77,1.56)$ \\
\hline
\end{tabular}

$-8-6-4-2024 \quad 468$

worse $<<$ functioning $\gg$ better
Role functioning

B (95\% Cl)

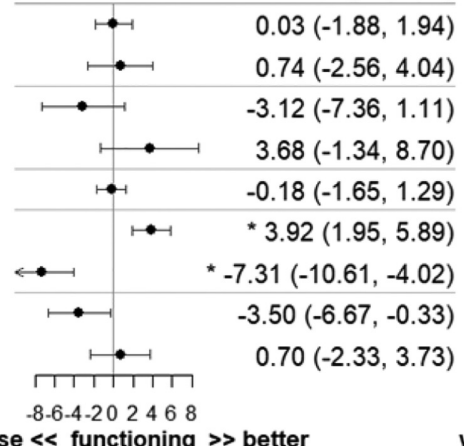

worse $<<$ functioning $\gg$ better

\section{Emotional functioning}

$\mathrm{B}(95 \% \mathrm{Cl})$

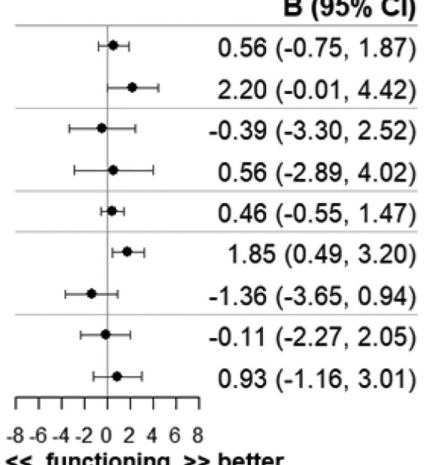

worse $<$ functioning $\gg$ better
Social functioning

B $(95 \% \mathrm{Cl})$

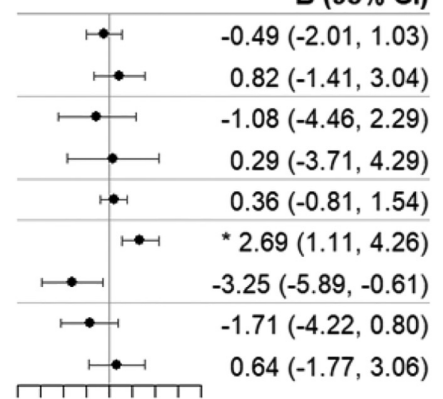

$-8-6-4-2022468$

worse $<<$ functioning $\gg$ better
Fatigue

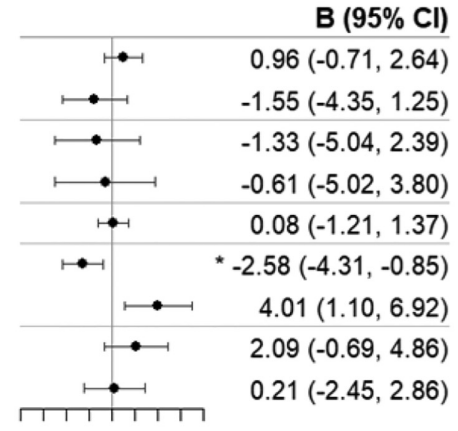

$-8-6-4-20202468$

less $\ll<$ fatigue $\gg$ more

FIGURE 2 Regression coefficients (B) and 95\% confidence intervals (95\% CI) of cross-sectional linear regression analyses of log2-transformed biomarker concentrations with aspects of health-related quality of life 6 months after colorectal cancer diagnosis. Analyses were adjusted for sex, age, cohort (COLON, EnCoRe, ColoCare), neo-adjuvant therapy (yes/no), adjuvant therapy (yes/no), cancer stage (I, II, III), any supplement use (yes/no), total energy intake (kcal/day), alcohol intake (gr/day), BMI $\left(\mathrm{kg} / \mathrm{m}^{2}\right)$, adherence to physical activity guidelines (yes/no), creatinine ( $\left.\mu \mathrm{mol} / \mathrm{L}\right)$, and cotinine $(\mathrm{nmol} / \mathrm{L}) .{ }^{*} \mathrm{Statistically}$ significant after FDR adjustment for multiple testing. Abbreviations: COLON, COlorectal cancer: Longitudinal, Observational study on Nutritional and lifestyle factors that influence colorectal tumour recurrence, survival and quality of life; EnCoRe, Energy for life after ColoRectal cancer; FDR, false discovery rate; $\mathrm{HKr}$, hydroxykynurenine ratio; QoL, quality of life.

\section{Multivariable regression analyses}

At 6 months after diagnosis, a doubling in the PLP concentration was statistically significantly associated with better physical functioning $(\beta, 2.55 ; 95 \% \mathrm{CI}: 1.31,3.80)$, role functioning $(\beta$, $3.92 ; 95 \% \mathrm{CI}: 1.95,5.89)$, and social functioning $(\beta, 2.69$; $95 \% \mathrm{CI}: 1.11,4.26)$, and decreased fatigue $(\beta,-2.58 ; 95 \% \mathrm{CI}$ :
$-4.31,-0.85)$, as analyzed in the cross-sectional confounderadjusted models after adjustment for multiple testing (Figure 2). Furthermore, statistically significant associations were observed for a doubling in the $\mathrm{HKr}$ concentration, the inverse marker of vitamin $\mathrm{B} 6$, and reduced global quality of life $(\beta,-3.62$; $95 \% \mathrm{CI}:-5.88,-1.36)$, worse physical functioning $(\beta,-5.01$; $95 \% \mathrm{CI}:-7.09,-2.94)$, and worse role functioning $(\beta,-7.31$; 
95\% CI: - 10.61, -4.02). No statistically significant associations were observed for the biomarkers of folate, folic acid, tHcy, methionine, riboflavin, cobalamin, and MMA with quality of life. In addition, no statistically significant associations were noted when modeling changes in biomarker concentrations between diagnosis and 6 months postdiagnosis (Figure 3). Comparable patterns were observed in minimally adjusted models, only adjusted for sex and age (Supplementary Table 2A and B).

The use of B-vitamin supplements was not associated with health-related quality of life 6 months postdiagnosis in the cross-sectional confounder-adjusted model (Table 3). Notably, participants who stopped using B-vitamin supplements after diagnosis reported higher fatigue $(\beta, 7.1 ; 95 \%$ CI: $2.9,11.2)$ in comparison to nonusers.

When outliers were excluded from the analyses, regression coefficients of PLP and HKr in relation to the quality of life outcomes were slightly attenuated, yet conclusions remained the same, as no notable changes were observed in the abovementioned associations (data not shown). In stratified analyses, individual cohorts showed associations that were in the same direction as the overall association, and associations within ColoCare were statistically significant less often than those within COLON and EnCoRe (data not shown). Further, when the inflammatory marker KTR was added to the models of PLP, regression coefficients were slightly attenuated, with $\beta$ values of 1.06 (95\% CI: $-0.33,2.46)$ for global quality of life, 1.81 (95\% CI: $0.54,3.09)$ for physical functioning, 3.04 (95\% CI: $1.02,5.06)$ for role functioning, 2.11 (95\% CI: $0.71,3.52)$ for emotional functioning, 1.42 (95\% CI: $-0.04,2.89)$ for cognitive functioning, 2.56 (95\% CI: 0.93, 4.18) for social functioning, and -2.26 (95\% CI: $-4.05,-0.48$ for fatigue. Lastly, doseresponse relationships were observed for the associations of deficient (PLP $<20 \mathrm{nmol} / \mathrm{L} ; n=92$ ), suboptimal (PLP 20-30 $\mathrm{nmol} / \mathrm{L} ; n=204)$, and adequate (PLP $>30 \mathrm{nmol} / \mathrm{L} ; n=791$ ) levels of vitamin B6 with global quality of life and physical, role, and social functioning (Figure 4).

\section{Discussion}

The current study aimed to investigate whether B-vitamin biomarkers and B-vitamin supplement use were associated with quality of life in survivors of CRC, both cross-sectionally at 6 months postdiagnosis and through the modeling of changes between diagnosis and 6 months postdiagnosis. Higher circulating concentrations of PLP were cross-sectionally associated with better physical, role, and social functioning, as well as with reduced fatigue, at 6 months postdiagnosis. In addition, higher $\mathrm{HKr}$, a novel and inverse marker for vitamin B6 status (26), was associated with decreased global quality of life and worse physical and role functioning. Dose-response relations were observed when deficient, suboptimal, and adequate levels of vitamin B6 were modeled in relation to global quality of life and physical, role, and social functioning at 6 months postdiagnosis. Only minimal changes were observed in the biomarker concentrations between diagnosis and 6 months postdiagnosis, which may explain the absence of associations between changes in biomarker concentrations and quality of life. In addition, no cross-sectional associations were found between B-vitamin supplement use and quality of life, yet participants who stopped using B-vitamin supplements after diagnosis reported higher fatigue compared to nonusers.

Vitamin B6 status emerged from the current analyses as a potential determinant of quality of life after a diagnosis of $\mathrm{CRC}$, while biomarkers of folate, riboflavin, and vitamin B12 were unrelated to quality of life. In previous studies, specific focus has been on vitamin B6 in relation to the risk of CRC (41) and, to a lesser extent, to survival (42). Associations of vitamin B6 with quality of life after diagnosis of cancer have previously been investigated in relation to specific conditions, but not in CRC survivors specifically. A systematic review including 5 intervention studies concluded that vitamin B6 supplementation may have beneficial effects on the prevention of hand-foot syndrome (43). A mini review on B vitamins in general showed that it is unclear to what extent $\mathrm{B}$ vitamins play a role in chemotherapy-induced peripheral neuropathy and whether supplementation may be an option for treatment (44). Further, hyperhomocysteinemia has previously been associated with the development of postoperative cognitive decline in surgical oncology patients (45). All in all, the results of the current study add to the existing literature on B vitamins and aspects of quality of life, and provide new insights in the association of vitamin B6 with fatigue and quality of life, specifically in CRC survivors.

The observed associations suggest that elevated vitamin B6 concentrations may be beneficial for quality of life after a CRC diagnosis. Hypothetically, PLP concentrations can be increased relatively easily through the consumption of foods high in vitamin B6 or through the use of vitamin B6 supplements. In our analyses, it was not possible to model vitamin B6 supplement use separately, because the vitamin is often part of vitamin complexes and is rarely used as a single supplement. We noted an association between participants who stopped using B vitamins and increased fatigue. The analyses provide insufficient evidence for the formulation of advice on the use of vitamin B6 for CRC survivors. Moreover, vitamin B6 status may be a reflection of the general nutritional state of individuals, and the adjustments for total energy intake and BMI may not have been sufficient to account for this. Thus, no firm conclusions can be drawn based on the observations of this study. Nevertheless, the data give important directions for future research on vitamin B6 and quality of life outcomes.

Potential confounding by inflammation was evaluated for the observed statistically significant associations of vitamin B6 with quality of life. As circulating PLP concentrations are known to be low in inflammatory conditions $(38,39,46)$, and because inflammation has been related to quality of life (47), the inflammatory markers CRP and KTR were added to the models to evaluate whether associations could be confounded by inflammation. Although the regression coefficients were slightly attenuated, further research is needed to evaluate the mechanistic role of inflammation in the association between $\mathrm{B}$ vitamins and aspects of quality of life and whether inflammation may influence the association as a possible confounder or mediator.

The ColoCare cohort seemed to differ in comparison to the COLON and EnCoRe cohorts with regard to quality of life, biomarker concentrations, and B-vitamin supplement use. The proportion of ColoCare participants with clinically relevant problems in quality of life and functioning was highest and on average the ColoCare participants scored lower on quality of life while the COLON and EnCoRe participants scored 


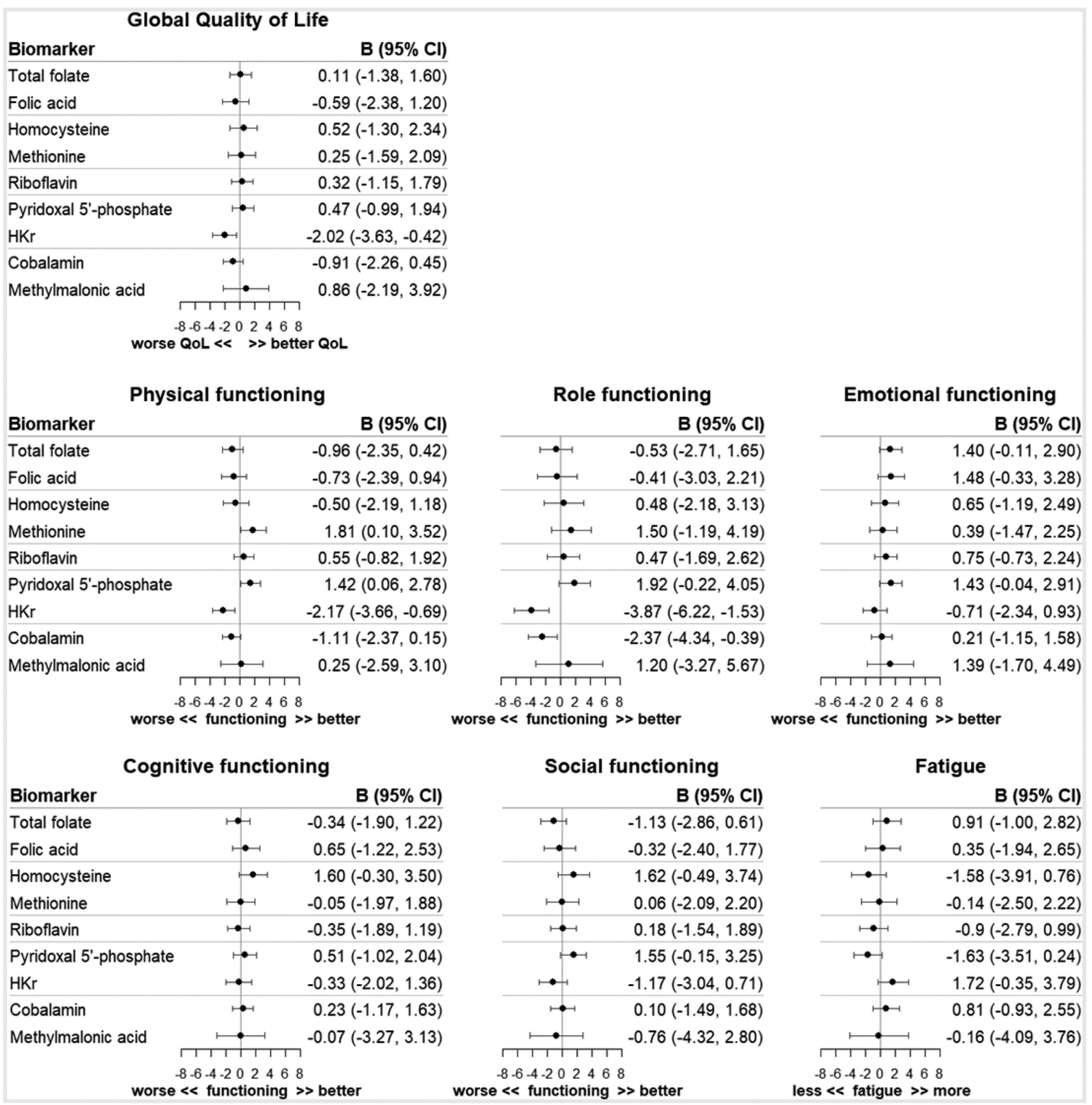

FIGURE 3 Regression coefficients (B) and 95\% confidence intervals (95\% CI) of linear regression analyses of standardized changes in biomarker concentrations (concentration at 6 months - concentration at diagnosis) with aspects of health-related quality of life 6 months after colorectal cancer diagnosis. Analyses were adjusted for sex, age, cohort (COLON, EnCoRe, ColoCare), neo-adjuvant therapy (yes/no), adjuvant therapy (yes/no), cancer stage (I, II, III), any supplement use (yes/no), biomarker concentration at diagnosis, total energy intake (kcal/day), alcohol intake (gr/day), BMI (kg/m²), adherence to physical activity guidelines $(\mathrm{yes} / \mathrm{no})$, creatinine $(\mu \mathrm{mol} / \mathrm{L})$, and cotinine $(\mathrm{nmol} / \mathrm{L})$. Abbreviations: COLON, COlorectal cancer: Longitudinal, Observational study on Nutritional and lifestyle factors that influence colorectal tumour recurrence, survival and quality of life; EnCoRe, Energy for life after ColoRectal cancer; FDR, false discovery rate; HKr, hydroxykynurenine ratio; QoL, quality of life.

higher, compared to reference data of CRC patients (stage IIV) with similar ages (48). When stratified by tumor location, ColoCare scoring was still significantly lower than COLON and EnCoRe scoring. Other clinical factors, such as the presence of a stoma, and/or cultural factors may be underlying these differences, although ColoCare participants also scored lower on all subscales of quality of life compared to 439 CRC patients from Germany using the same questionnaire (49). Global quality of life scores were similar, whereas physical functioning and fatigue scores were lower, compared to previous analyses from the ColoCare study measured at diagnosis and after 12 months (50). However, timing may be crucial, as patients likely experience 
TABLE 3 Results of cross-sectional linear regression analyses of B-vitamin supplement use 6 months after diagnosis and of associations of changes in B-vitamin supplement use between diagnosis and 6 months after diagnosis with health-related quality of life

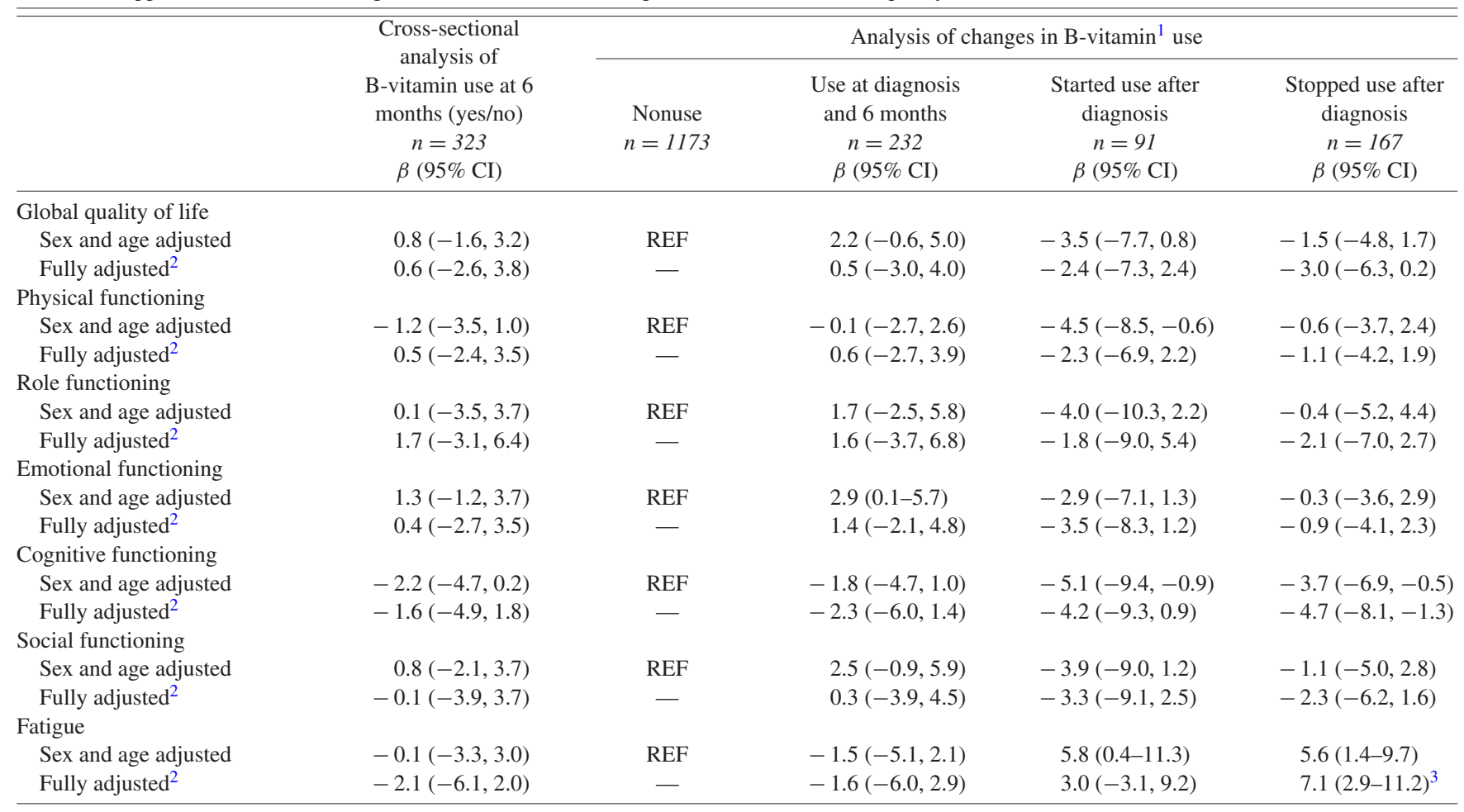

${ }^{1}$ B-vitamin supplement use was defined as the use of dietary supplements containing at least 1 of the B vitamins: folic acid, vitamin B2, vitamin B6, or vitamin B12.

${ }^{2}$ Fully adjusted model: adjusted for sex, age, cohort (COLON, EnCoRe, ColoCare), neo-adjuvant therapy (yes/no), adjuvant therapy (yes/no), cancer stage (I, II, III), total energy intake ( $\mathrm{kcal} / \mathrm{day}), \mathrm{BMI}\left(\mathrm{kg} / \mathrm{m}^{2}\right)$, adherence to physical activity guidelines (yes/no), and any supplement use (yes/no).

${ }^{3}$ Statistically significant after FDR adjustment for multiple testing.

the lowest quality of life shortly after therapy. Further, ColoCare participants had higher total folate concentrations, which may be explained by the faster degradation of folate in plasma (COLON and EnCoRe) compared to serum samples (ColoCare). The higher proportion of rectum cancer patients and/or the lower proportion of supplement users in ColoCare could possibly explain the lower concentrations of PLP in ColoCare participants. All models were adjusted for confounding by cohort and, despite the differences in exposures and outcomes between cohorts, stratified analyses showed comparable associations across cohorts, thereby providing some degree of confidence that the observed associations were not strongly influenced by these differences.

An important strength of the current study is its novel and explorative character/design, as associations between biomarkers of B vitamins and quality of life have not been investigated in CRC patients before. Furthermore, data were obtained from a consortium of multiple prospective cohorts with comparable participant recruitment and data collection methods and a relatively high number of CRC patients, strengthening the generalizability of our findings. All biochemical analyses were carried out at a centralized laboratory, which delivered a broad panel of targeted and precise profiling of multiple B vitamins and complementary functional biomarkers.

The study also has limitations. First, the intake of B vitamins from diet and supplements could not be taken into account due to unavailability of details on the dietary B-vitamin intake and the exact amount of B-vitamin intake from supplements from all cohorts. Second, biomarker data could have been influenced by several factors, such as the inclusion of both fasting and nonfasting samples and the small number of COLON participants who may have still been undergoing chemotherapy at the time of the 6-month postdiagnosis measurement. However, these aspects could not be accounted for, as details on the fasting state were unavailable for all cohorts and the therapy end dates were unknown for COLON participants. Lastly, the observational nature of the study makes it difficult to differentiate between cause and effect.

In conclusion, the current study provides an important, novel lead regarding the multiple possible aspects with which to improve health-related quality of life after CRC. Vitamin B6 status was found to be associated with better quality of life, better functioning, and less fatigue in combined analyses of 3 European prospective studies. However, these associations are based on analyses of 2 time points with a 1-time measurement of the outcome, and no significant associations were observed for B-vitamin supplement use. Vitamin B6 needs to be investigated in future longitudinal studies with more repeated measurements to confirm our findings and to provide a stronger foundation for potential requirements of intervention studies in CRC patients. In addition, future studies should also consider the intake of $\mathrm{B}$ vitamins from diet and supplements to accurately estimate 
Global QoL

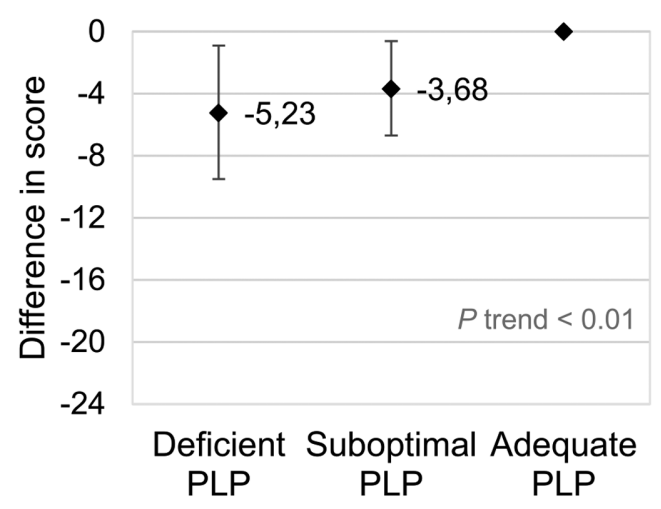

Role functioning

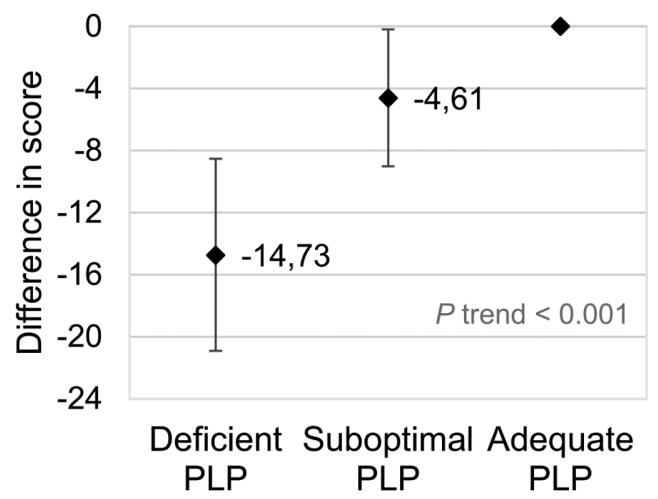

Physical functioning

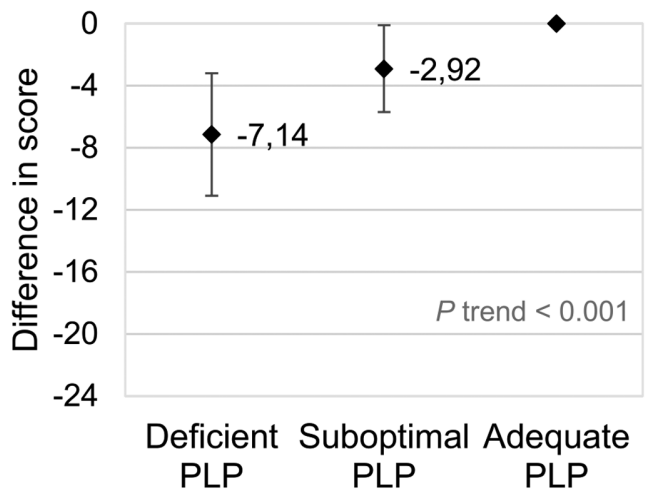

Social functioning

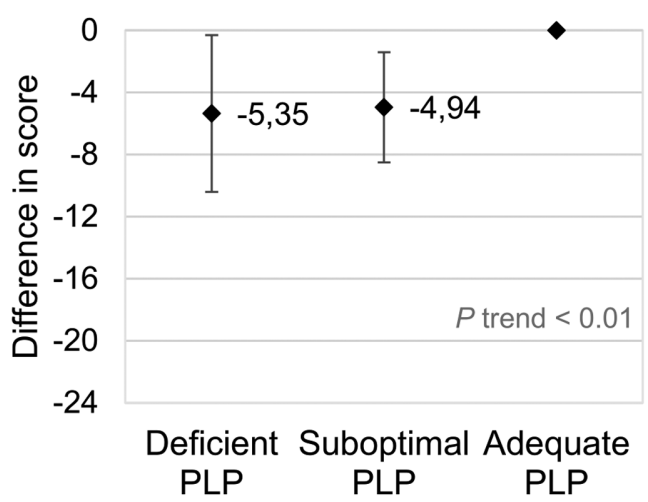

FIGURE 4 Dose-response relationships between vitamin B6 status, measured as PLP, and quality of life outcomes. Deficient, suboptimal, and adequate vitamin B6 levels were defined as serum/plasma PLP $<20 \mathrm{nmol} / \mathrm{L}(n=92), 20-30 \mathrm{nmol} / \mathrm{L}(n=204)$, and $>30 \mathrm{nmol} / \mathrm{L}(n=791)$, respectively. Abbreviations: PLP, pyridoxal 5'-phosphate; QoL, quality of life.

the total B-vitamin intake and further evaluate potential doseresponse relationships.

We thank all COLON, EnCoRe, and ColoCare participants; the COLON investigators at Wageningen University \& Research and the involved coworkers in the participating COLON hospitals; and all participants of the EnCoRe study and the health professionals in the 3 hospitals involved in the recruitment of participants of EnCoRe (Maastricht University Medical Center+, VieCuri Medical Center, and Zuyderland Medical Center). We thank the MEMIC center for data and information management and for facilitating the logistic processes and data management of our study. Finally, we thank the research dieticians and research assistants who are responsible for patient inclusion and follow-up, performing home visits, and data collection and processing. We thank the liquid biobank of the National Center for Tumor Diseases (NCT) for storing the ColoCare samples according to the standard operating procedures of the Biomaterialbank Heidelberg, and Lin Zielske, Anett Brendel, Renate Skatula, and Marita Wenzel for supporting biobanking at the NCT. In addition, we thank Rifraz Farook and Werner Diehl for their data management support and the ColoCare teamspecifically, Torsten Kölsch, Clare Abbenhardt-Martin, Susanne Jakob, and Judith Kammer-for patient recruitment and follow-up.

The authors' responsibilities were as follows - MPW, MJLB, EK, PMU, $\mathrm{CMU}$, AG: conceived of the project, developed the overall research plan, and provided study oversight; JLK, AJMRG, BG, DEK, FJBvD, SB, TG, ANH, RK, TL, JO, EHvR, AU, EW, HB, PS-K, SOB, MLGJ-H, ETPK, HKvH, JHWdW: conducted hands-on experiments and data collection; JLK, AJMRG, BG, DEK, FJBvD, SB, TG, ANH, RK, TL, JO, EHvR, AU, ØM,
EW, HB, PS-K, SOB, MLGJ-H, ETPK, HKvH, JHWdW, CMU: provided essential reagents or essential materials, including animals, constructs, databases, and so forth, necessary for the research; JLK, MJLB, MPW: analyzed the data or performed the statistical analysis and made a major contribution to writing the paper; MPW, MJLB, EK, PMU, CMU, AG: had primary responsibility for the final content; all authors: reviewed the manuscript critically, provided feedback, and read and approved the final manuscript.

Author disclosures: JLK is funded by Kankeronderzoekfonds Limburg as part of Health Foundation Limburg (Grant No. 00005739). EHvR is funded by the Wereld Kanker Onderzoek Fonds (WKOF), as part of the World Cancer Research Fund International grant program (grant number 2016/1620). ANH was supported by the Vanderbilt University Medical Center and the National Institutes of Health under Ruth L. Kirschstein National Research Service Award T32 HG008962 from the National Human Genome Research Institute. JO, JB, ANH, RK, TL, ARP, and CMU, who are investigators at the Huntsman Cancer Institute, were supported by grants from the National Institutes of Health/National Cancer Institute (U01CA206110, R01CA189184 and R01 CA207371 to CMU), the Stiftung LebensBlicke, and the Huntsman Cancer Foundation. CMU formally oversees research funded by several pharmaceutical companies as Executive Director of the Comprehensive Cancer Center at Huntsman Cancer Institute (Salt Lake City, UT); however, she does not direct those research efforts and has not received funding directly herself that would constitute a conflict to the current work. MJLB, AJMRG, BG, AU, DEK, SB, AB, HB, SOB, JC-C, FJBvD, PvD, TG, $\mathrm{NH}, \mathrm{HKvH}, \mathrm{MH}, \mathrm{MLGJ}-\mathrm{H}, \mathrm{ETPK}, \mathrm{FMK}, \mathrm{CIL}, \varnothing \mathrm{M}, \mathrm{MAS}, \mathrm{PS}-\mathrm{K}, \mathrm{ABU}, \mathrm{KV}$, EW, JHWdW, AG, PMU, EK, and MPW, no conflicts of interest. 


\section{Data Availability}

Data described in the manuscript, code book, and analytic code will not be made available because of restrictions in patient consent and Institutional Review Board approval, as well as ongoing analyses.

\section{References}

1. Drury A, Payne S, Brady AM. Cancer survivorship: Advancing the concept in the context of colorectal cancer. Eur J Oncol Nurs 2017;29:135-47.

2. Arnold M, Sierra MS, Laversanne M, Soerjomataram I, Jemal A, Bray F. Global patterns and trends in colorectal cancer incidence and mortality. Gut 2017;66(4):683-91.

3. Siegel RL, Miller KD, Goding Sauer A, Fedewa SA, Butterly LF, Anderson JC, Cercek A, Smith RA, Jemal A. Colorectal cancer statistics, 2020. CA Cancer J Clin 2020;70(3):145-164.

4. Brouwer NPM, Bos A, Lemmens V, Tanis PJ, Hugen N, Nagtegaal ID, de Wilt JHW, Verhoeven RHA. An overview of 25 years of incidence, treatment and outcome of colorectal cancer patients. Int $\mathrm{J}$ Cancer 2018;143(11):2758-66.

5. Mason JB, Tang SY. Folate status and colorectal cancer risk: A 2016 update, Mol Aspects Med 2017;53:73-9.

6. Miller JW, Ulrich CM. Folic acid and cancer-Where are we today? Lancet North Am Ed 2013;381(9871):974-6.

7. Bower JE. Cancer-related fatigue-Mechanisms, risk factors, and treatments. Nat Rev Clin Oncol 2014;11(10):597-609.

8. Husson O, Mols F, van de Poll-Franse L, de Vries J, Schep G, Thong MS. Variation in fatigue among 6011 (long-term) cancer survivors and a normative population: A study from the population-based PROFILES registry. Support Care Cancer 2015;23(7):2165-74.

9. Koole JL, Bours MJL, Breedveld-Peters JJL, van Roekel EH, Breukink SO, Janssen-Heijnen MLG, Vogelaar FJ, Aquarius M, Keulen E, Stoot $\mathrm{J}$, et al. Is dietary supplement use longitudinally associated with fatigue in stage I-III colorectal cancer survivors? Clin Nutr 2020;39(1):234-41.

10. Werbach MR. Nutritional strategies for treating chronic fatigue syndrome. Altern Med Rev 2000;5(2):93-108.

11. Scholten AM, Vermeulen E, Dhonukshe-Rutten RAM, Verhagen T, Visscher A, Olivier A, Timmer L, Witteman BJM. Surplus vitamin B12 use does not reduce fatigue in patients with irritable bowel syndrome or inflammatory bowel disease: A randomized double-blind placebocontrolled trial. Clin Nutr ESPEN 2018;23:48-53.

12. Araujo JR, Martel F, Borges N, Araujo JM, Keating E. Folates and aging: Role in mild cognitive impairment, dementia and depression. Ageing Res Rev 2015;22:9-19.

13. Balk EM, Raman G, Tatsioni A, Chung M, Lau J, Rosenberg IH. Vitamin B6, B12, and folic acid supplementation and cognitive function: A systematic review of randomized trials. Arch Intern Med 2007;167(1):21-30.

14. Lehert P, Villaseca P, Hogervorst E, Maki PM, Henderson VW. Individually modifiable risk factors to ameliorate cognitive aging: A systematic review and meta-analysis. Climacteric 2015;18(5):678-89.

15. Morris MS, Jacques PF, Rosenberg IH, Selhub J. Circulating unmetabolized folic acid and 5-methyltetrahydrofolate in relation to anemia, macrocytosis, and cognitive test performance in American seniors. Am J Clin Nutr 2010;91(6):1733-44.

16. Troen AM, Mitchell B, Sorensen B, Wener MH, Johnston A, Wood B, Selhub J, McTiernan A, Yasui Y, Oral E, et al. Unmetabolized folic acid in plasma is associated with reduced natural killer cell cytotoxicity among postmenopausal women. J Nutr 2006;136(1):189-94.

17. Rolita L, Holtzer R, Wang C, Lipton RB, Derby CA, Verghese J. Homocysteine and mobility in older adults. J Am Geriatr Soc 2010;58(3):545-50.

18. van Schoor NM, Swart KM, Pluijm SM, Visser M, Simsek S, Smulders Y, Lips P. Cross-sectional and longitudinal association between homocysteine, vitamin B12 and physical performance in older persons. Eur J Clin Nutr 2012;66(2):174-81.

19. Bender A, Hagan KE, Kingston N. The association of folate and depression: A meta-analysis. J Psychiatr Res 2017;95:9-18.

20. Marian MJ. Dietary supplements commonly used by cancer survivors: Are there any benefits? Nutr Clin Pract 2017;32(5):607-27.
21. Du M, Luo H, Blumberg JB, Rogers G, Chen F, Ruan M, Shan Z, Biever E, Zhang FF. Dietary supplement use among adult cancer survivors in the United States. J Nutr 2020;150(6):1499-508.

22. Winkels RM, Heine-Broring RC, van Zutphen M, van Harten-Gerritsen S, Kok DE, van Duijnhoven FJ, Kampman E. The COLON study: Colorectal cancer: Longitudinal, observational study on nutritional and lifestyle factors that may influence colorectal tumour recurrence, survival and quality of life. BMC Cancer 2014;14:374.

23. van Roekel EH, Bours MJ, de Brouwer CP, Ten Napel H, Sanduleanu S, Beets GL, Kant IJ, Weijenberg MP. The applicability of the international classification of functioning, disability, and health to study lifestyle and quality of life of colorectal cancer survivors. Cancer Epidemiol Biomark Prev 2014;23(7):1394-405.

24. Ulrich CM, Gigic B, Bohm J, Ose J, Viskochil R, Schneider M, Colditz GA, Figueiredo JC, Grady WM, Li CI, et al. The ColoCare study: A paradigm of transdisciplinary science in colorectal cancer outcomes. Cancer Epidemiol Biomark Prev 2019;28(3): 591-601.

25. Hannisdal R, Ueland PM, Eussen SJ, Svardal A, Hustad S. Analytical recovery of folate degradation products formed in human serum and plasma at room temperature. J Nutr 2009;139(7):1415-8.

26. Ulvik A, Midttun O, McCann A, Meyer K, Tell G, Nygard O, Ueland PM. Tryptophan catabolites as metabolic markers of vitamin B-6 status evaluated in cohorts of healthy adults and cardiovascular patients. Am J Clin Nutr 2020;111(1):178-86.

27. Hannisdal R, Ueland PM, Svardal A. Liquid chromatography-tandem mass spectrometry analysis of folate and folate catabolites in human serum. Clin Chem 2009;55(6):1147-54.

28. Midttun O, Hustad S, Ueland PM. Quantitative profiling of biomarkers related to B-vitamin status, tryptophan metabolism and inflammation in human plasma by liquid chromatography/tandem mass spectrometry. Rapid Commun Mass Spectrom 2009;23(9):1371-9.

29. Midttun $\varnothing$, Kvalheim G, Ueland PM. High-throughput, low-volume, multianalyte quantification of plasma metabolites related to onecarbon metabolism using HPLC-MS/MS. Anal Bioanal Chem 2013;405(6):2009-17.

30. Midttun O, McCann A, Aarseth O, Krokeide M, Kvalheim G, Meyer $\mathrm{K}$, Ueland PM. Combined measurement of 6 fat-soluble vitamins and 26 water-soluble functional vitamin markers and amino acids in $50 \mu \mathrm{L}$ of serum or plasma by high-throughput mass spectrometry. Anal Chem 2016;88(21):10427-36.

31. Kelleher BP, Broin SD. Microbiological assay for vitamin B12 performed in 96-well microtitre plates. J Clin Pathol 1991;44(7):592-5.

32. Meyer K, Ueland PM. Targeted quantification of C-reactive protein and cystatin $\mathrm{C}$ and its variants by immuno-MALDI-MS. Anal Chem 2014;86(12):5807-14.

33. Aaronson NK, Ahmedzai S, Bergman B, Bullinger M, Cull A, Duez NJ, Filiberti A, Flechtner H, Fleishman SB, de Haes JC, et al. The European Organization for Research and Treatment of Cancer QLQC30: A quality-of-life instrument for use in international clinical trials in oncology. J Natl Cancer Inst 1993;85(5):365-76.

34. Fayers PM, Aaronson NK, Bjordal K, Groenvold M, Curran D, Bottomley A; on behalf of the European Organisation for Research and Treatment of Cancer (EORTC) Quality of Life Group. The EORTC QLQ-C30 scoring manual. 3rd ed. Brussels, Belgium: European Organisation for Research and Treatment of Cancer; 2001.

35. Giesinger JM, Loth FLC, Aaronson NK, Arraras JI, Caocci G, Efficace F, Groenvold M, van Leeuwen M, Petersen MA, Ramage J, et al. Thresholds for clinical importance were established to improve interpretation of the EORTC QLQ-C30 in clinical practice and research. J Clin Epidemiol 2020;118:1-8.

36. Miller MF, Bellizzi KM, Sufian M, Ambs AH, Goldstein MS, BallardBarbash R. Dietary supplement use in individuals living with cancer and other chronic conditions: A population-based study. J Am Diet Assoc 2008;108(3):483-94.

37. Benjamini Y, Hochberg Y. Controlling the false discovery rate: A practical and powerful approach to multiple testing. J R Stat Soc Series B Stat Methodol 1995;57(1):289-300.

38. Kiblawi R, Holowatyj AN, Gigic B, Brezina S, Geijsen A, Ose J, Lin T, Hardikar S, Himbert C, Warby CA, et al. One-carbon metabolites, B vitamins and associations with systemic inflammation and angiogenesis biomarkers among colorectal cancer patients: Results from the ColoCare Study. Br J Nutr 2020;123(10): 1187-200. 
39. Ueland PM, McCann A, Midttun O, Ulvik A. Inflammation, vitamin B6 and related pathways. Mol Aspects Med 2017;53:10-27.

40. Stover PJ, Field MS. Vitamin B-6. Adv Nutr 2015;6(1):132-3.

41. Larsson SC, Orsini N, Wolk A. Vitamin B6 and risk of colorectal cancer: A meta-analysis of prospective studies. JAMA 2010;303(11): 1077-83.

42. Je Y, Lee JE, Ma J, Zhang X, Cho E, Rosner B, Selhub J, Fuchs CS, Meyerhardt J, Giovannucci E. Prediagnostic plasma vitamin B6 (pyridoxal 5'-phosphate) and survival in patients with colorectal cancer. Cancer Causes Control 2013;24(4):719-29.

43. Chen M, Zhang L, Wang Q, Shen J. Pyridoxine for prevention of handfoot syndrome caused by chemotherapy: A systematic review. PLOS One 2013;8(8):e72245.

44. Schloss J, Colosimo M. B vitamin complex and chemotherapyinduced peripheral neuropathy. Curr Oncol Rep 2017;19(12): 76.

45. Weerink LBM, van Leeuwen BL, Gernaat SAM, Absalom AR, Huisman MG, van der Wal-Huisman H, Izaks GJ, de Bock GH. Vitamin status and the development of postoperative cognitive decline in elderly surgical oncologic patients. Ann Surg Oncol 2018;25(1): $231-8$.
46. Abbenhardt C, Miller JW, Song X, Brown EC, Cheng TY, Wener MH, Zheng Y, Toriola AT, Neuhouser ML, Beresford SA, et al. Biomarkers of one-carbon metabolism are associated with biomarkers of inflammation in women. J Nutr 2014;144(5):714-21.

47. de Almeida Roediger M, de Fátima Nunes Marucci M, Duim EL, Santos JLF, de Oliveira Duarte YA, de Oliveira C. Inflammation and quality of life in later life: findings from the health, well-being and aging study (SABE). Health Qual Life Outcomes 2019;17(1):26.

48. Scott NW, Fayers P, Aaronson NK, Bottomley A, de Graeff A, Groenvold M, Gundy C, Koller M, Petersen MA, Sprangers MAG. EORTC QLQ-C30 reference values manual. 2nd ed. Brussels, Belgium: EORTC Quality of Life Group; 2008.

49. Jansen L, Herrmann A, Stegmaier C, Singer S, Brenner H, Arndt V. Health-related quality of life during the 10 years after diagnosis of colorectal cancer: A population-based study. J Clin Oncol 2011;29(24):3263-9.

50. Gigic B, Boeing H, Toth R, Bohm J, Habermann N, Scherer D, Schrotz-King P, Abbenhardt-Martin C, Skender S, Brenner H, et al. Associations between dietary patterns and longitudinal quality of life changes in colorectal cancer patients: The ColoCare study. Nutr Cancer 2018;70(1):51-60. 